\title{
Tao Brush
}

\author{
Ashok Kumar ${ }^{1}$
}

Published online: 13 May 2017

(C) Federation of Obstetric \& Gynecological Societies of India 2017

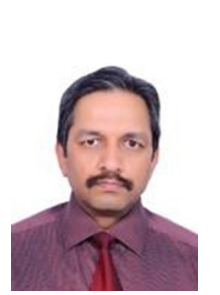

\begin{abstract}
About the Reviewer
Ashok Kumar studied at the All India Institute of Medical Sciences, New Delhi and obtained his Ph.D. from Tokyo Women's Medical University, Japan. He has a special interest in maternal health and has published research papers on thyroid hormonal changes during pregnancy, calcium supplementation during pregnancy for preventing hypertensive disorders (included in a Cochrane review, 2010), hepatitis E (reviewed in Obstetrics and Gynecology Survey) and C during pregnancy, latent celiac disease in reproductive performance, dydrogesterone in recurrent pregnancy loss (included in the European Progestin Club guidelines, 2015) and preeclampsia. He participated in the international, multicentre 'CORONIS' trial that aimed to assess cesarean section surgical techniques and maternal morbidity. He has delivered Yuva FOGSI Oration 'Thyroid disorders during pregnancy' at North Zone Yuva FOGSI Conference, 2004 and Golden Jubilee Commemoration Award Lecture of National Academy of Medical Sciences (India) 2013 and won FOGSI-Corion award 2008 for best scientific work. Dr Kumar has authored the book 'Essentials of Gynaecology' and edited the books: Common Medical Disorders in Obstetrics and Drugs in Obstetrics and
\end{abstract} Gynecology.

The Tao brush is a small flexible brush used in TruTest ${ }^{\circledR}$ Endometrial Biopsy. Endometrial tissue to a depth of $1.5-2 \mathrm{~mm}$ can be collected. It gently brushes the entire inside of the uterus so as to gather a more complete sampling of the endometrium and is less painful than the traditional method [1]. It covers a wide area of the endometrial surface. Therefore, small lesions are less likely

Ashok Kumar is a Director Professor of Department of Obstetrics and Gynaecology in Maulana Azad Medical College and Lok Nayak Hospital, New Delhi 110002, India.

\section{Ashok Kumar}

ash64kr@yahoo.com

1 Director Professor, Department of Obstetrics and Gynaecology, Maulana Azad Medical College and Lok Nayak Hospital, New Delhi 110002, India to be missed. It is able to detect endometrial adenocarcinoma also.

\section{Parts of Tao Brush}

The total length is about $26 \mathrm{~cm}$; brush head length is about $3-3.5 \mathrm{~cm}$ and diameter of 9 French (Fig. 1). It has a covering sheath which protects the brush from collecting any contaminating tissue from the cervix. A sound mark is provided on the brush to indicate depth of $6.5 \mathrm{~cm}$.

\section{Procedure}

In a simple in-office procedure, the patient is made to lie down in dorsal lithotomy position. After routine pelvic examination, the brush is inserted into the uterus. The 


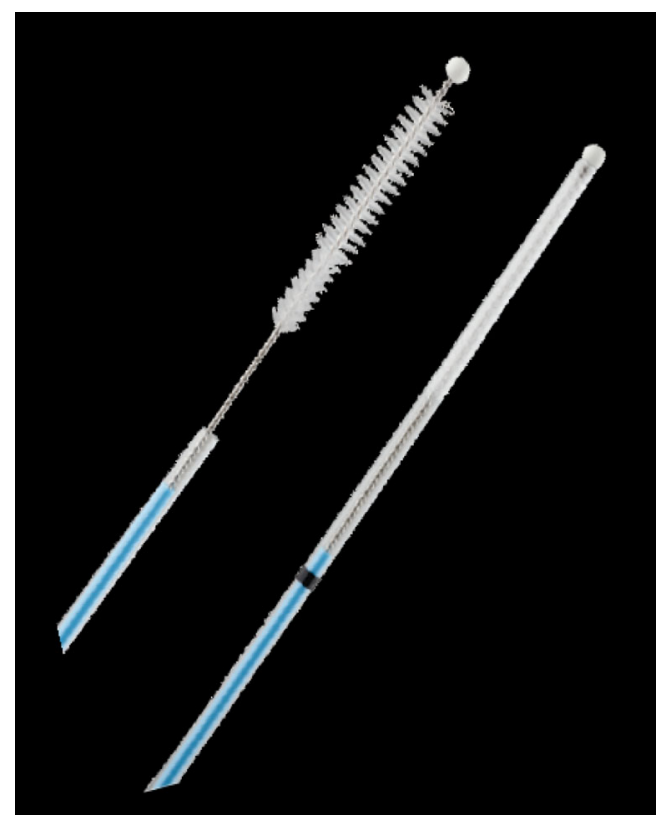

Fig. 1 Tao Brush with covering sheath

covering sheath will protect the brush from collecting any contaminating tissue from the cervix. Once the brush is in place, the sheath is removed. It is then rotated 4-5 times by a simple watch-winding motion to collect tissue from the entire endometrium. The sheath is then replaced, ensuring that the endometrial tissue retained on the brush. The brush is removed and placed directly in the fixative solution. The entire procedure takes approximately $30 \mathrm{~s}$.

\section{Uses}

The Tao Brush is a patented device, FDA approved, class II instrument basically used for endometrial histology and cytology sampling.

The main screening devices available for endometrial carcinoma are aspiration devices (such as the Vabra aspirator, Pipelle and Tao Brush). Among these devices, the Tao Brush is the most promising endometrial sampler for screening for endometrial lesions [2].

In a comparative study of 220 women with abnormal uterine bleeding, endometrial sampling was done before cervical dilatation using Tao Brush followed by conventional dilatation and curettage (D\&C). All (100\%) of samples obtained by conventional D\&C, and $98.2 \%$ of the samples obtained by Tao Brush were adequate for histopathology examination. Tao Brush showed 100\% sensitivity, $100 \%$ specificity, $100 \%$ predictive values and accuracy for diagnosing proliferative and secretory endometrium, endometrial hyperplasia, endometrial carcinoma. It also showed $86.7 \%$ sensitivity, $100 \%$ specificity, $100 \%$ positive predictive value (PPV) and $99 \%$ negative predictive value (NPV) and accuracy for diagnosing endometritis (no significant difference compared to conventional D\&C) and $77.8 \%$ sensitivity, $100 \%$ specificity, $100 \% \mathrm{PPV}$ and 99\% NPV and accuracy for diagnosing endometrial polyps (no significant difference compared to conventional D\&C) [3]. Another study reported $95.5 \%$ sensitivity rate and $100 \%$ specificity for detection of endometrial cancer [4]. Along with early detection of endometrial cancer, the TruTest also tests for chlamydia, gonorrhea and human papilloma virus.

In postmenopausal women, Tao Brush sampling is superior to Pipelle endometrial biopsy in obtaining an adequate sample [5]. It was found to be up to one and half times less painful than Pipelle [1].

The Tao Brush is a better tolerated invasive device for collection of endometrial tissue. It minimizes sample contamination from the endocervix and has high sensitivity and specificity for the detection of endometrial hyperplasia and endometrial carcinoma.

\section{References}

1. Yang G, Wan LS. Endometrial biopsy using the Tao Brush method. A study of 50 women in a general gynecologic practice. J Reprod Med. 2000;45(2):109-14.

2. Du J, Li Y, Lv S, et al. Endometrial sampling devices for early diagnosis of endometrial lesions. J Cancer Res Clin Oncol. 2016;142:2515-22.

3. Abdelazim IA, Abdelrazak KM, Elbiaa AA, et al. Accuracy of endometrial sampling compared to conventional dilatation and curettage in women with abnormal uterine bleeding. Arch Gynecol Obstet. 2015;291(5):1121-6.

4. Del Priore G, Williams R, Harbatkin CB, et al. Endometrial brush biopsy for the diagnosis of endometrial cancer. J Reprod Med. 2001;46(5):439-43.

5. Williams A, Brechin S, Porter A, et al. Factors affecting adequacy of Pipelle and Tao Brush endometrial sampling. BJOG. 2008;115:1028-36. 